\title{
RESEARCH HIGHLIGHT Staged profiling of sperm development in sync
}

\author{
Navin B. Ramakrishna $\mathbb{D}^{1,2}$ and M. Azim Surani $\mathbb{D}^{1,3}$ \\ Cell Research (2018) 28:965-966; https://doi.org/10.1038/s41422-018-0088-5
}

\begin{abstract}
By synchronizing the first wave of spermatogenesis, Chen and colleagues profile gene levels and regulation dynamics in 20 stages of sperm development. From this, newfound molecular signatures of sperm maturation are unearthed and validated.
\end{abstract}

In humans, 250 million sperm are made daily, taking $\sim 74$ days to reach maturation involving a sequential process of differentiation, mitotic proliferation and meiosis, culminating in spermiogenesis. ${ }^{1}$ Sperm development in the mouse, while taking half the time, is no less complex. Understanding how this protracted process of spermatogenesis proceeds is of great interest, considering that defects in this process lead to infertility. In a recent paper in Cell Research, Chen et al. describe their strategy to meticulously purify and profile the development of sperm, crucially using a synchronized mouse model. ${ }^{2}$ This analysis has not only allowed for the characterization of gene dynamics during maturation, but has also led to the discovery and validation of previously uncharacterized stage-specific regulators and surface markers.

Spermatogenesis is comprehensively characterized at the morphological and histological levels, featuring 39 distinct morphological steps in total. ${ }^{3}$ Commencing from 3 days postpartum (dpp) in seminiferous tubules, spermatogonia at the base divide and differentiate, followed by a prolonged period of meiosis giving rise to haploid spermatids which elongate to form mature sperm in the tubule lumen. While RNA-sequencing studies of purified cells have revealed critical underlying molecular regulatory processes, the heterogeneity and asynchrony of germ cells in such bulk approaches have limited the stage-specific molecular characterization of sperm development.

Modern advances have overcome the characterization of heterogeneous cell populations with the ability to profile gene levels in individual cells via single-cell mRNA sequencing (scRNAseq). ${ }^{4}$ This technique has been used to reveal the molecular dynamics underlying differentiation in a variety of developing tissues. scRNA-seq has recently been used to profile spermatogenesis in the adult mouse testes, highlighting the progressive continuity of differentiation of germ cells. ${ }^{5}$ However, spermatogonial sub-populations were under-represented in the previous study due to their low abundance in highly heterogeneous adult testes.

In the new study, Chen et al. tackle the problem of capturing low-abundant sub-populations by synchronizing the first wave of spermatogenesis in juvenile mice, which otherwise exhibit considerable asynchrony. ${ }^{6}$ This was achieved by using a Retinoic Acid (RA) synthesis inhibitor at $2 \mathrm{dpp}$ to arrest the onset of spermatogenesis, followed by RA treatment. Synchronized germ cells were collected in 20 stages up till round spermatid development, oversampling especially the more poorlycharacterized stages of sperm development (Fig. 1a). In particular, germ cells at each time point were collected with a combination of FACS and the Unipick single-cell acquisition system based on gene reporter expression, DNA content and cell size. The authors stringently validated each stage for homogeneity and synchrony using electron and fluorescence microscopy.

Gene expression profiles of cells of each stage were highly correlated to each other, further validating the high homogeneity of purified cells. Interestingly, cells were grouped into seven clusters despite coming from 20 different developmental stages. The most significant cluster contained morphologically different spermatogonial cells, suggesting a high degree of similarity at the mRNA level. On the other hand, the round spermatids sampled at four stages fell into three distinct clusters, reflecting the high degree of differences during spermiogenesis. Of note, the lack of a continuous global trajectory in clustering analysis, as seen previously in the adult testes, most likely stems from the enrichment of cells from only specific time points, an apparent trade-off for omitting rare sub-populations. ${ }^{5}$

The most surprising observation was that the preleptotene spermatocytes in mid S-phase were found in different clusters, either grouping with the mitotic spermatogonial cluster or late Sphase preleptotene spermatocyte cluster, despite synchronous cell collection. While a gradual commitment to meiosis was generally observed, the enrichment of upregulated meiotic genes and downregulation of mitotic genes between early and midpreleptotene highly suggest that the mitotic-to-meiosis switch occurs then. This finding highlights the advantage of dense time point sampling to detect regulatory transitions.

The key strength of this work is in the identification of novel regulators and markers of spermatogenesis, highlighting the utility of data-rich scRNA-seq studies (Fig. 1b). Chen and colleagues found that the poorly characterized Fbxo47 gene was enriched in the early meiosis cluster, with strikingly similar gene expression patterns as known markers for DNA recombination. Male mice were sterile upon germline-specific Fbxo47 knockout, with spermatogenesis arrested at meiotic prophase l, suggesting a novel role for $\mathrm{FbxO47}$ in regulating recombination. In addition, Sox30, identified in transcription factor networks of the round spermatid clusters, was validated to be involved in spermiogenesis, with round spermatid arrest seen in the knockout, concurring with a recent study. ${ }^{7}$ Lastly, the authors identified CD63 as a potential differential surface marker for early $\left(\mathrm{CD}^{2} 3^{+}\right)$and late $\left(\mathrm{CD}^{-} 3^{-}\right)$round spermatids. Notably, the authors confirmed that sorted $\mathrm{CD}^{-} 3^{-}$round spermatids were more efficient in supporting blastocyst development via the artificial reproductive technique of intracytoplasmic round spermatid injection (ROSI) compared to

\footnotetext{
${ }^{1}$ Wellcome/Cancer Research UK Gurdon Institute, Tennis Court Road, Cambridge, CB2 1QN, UK; ${ }^{2}$ Department of Genetics, University of Cambridge, Downing Street, Cambridge, CB2 3EH, UK and ${ }^{3}$ Department of Physiology, Development and Neuroscience, University of Cambridge, Downing Street, Cambridge, CB2 3EG, UK

Correspondence: M. Azim Surani (a.surani@gurdon.cam.ac.uk)
} 

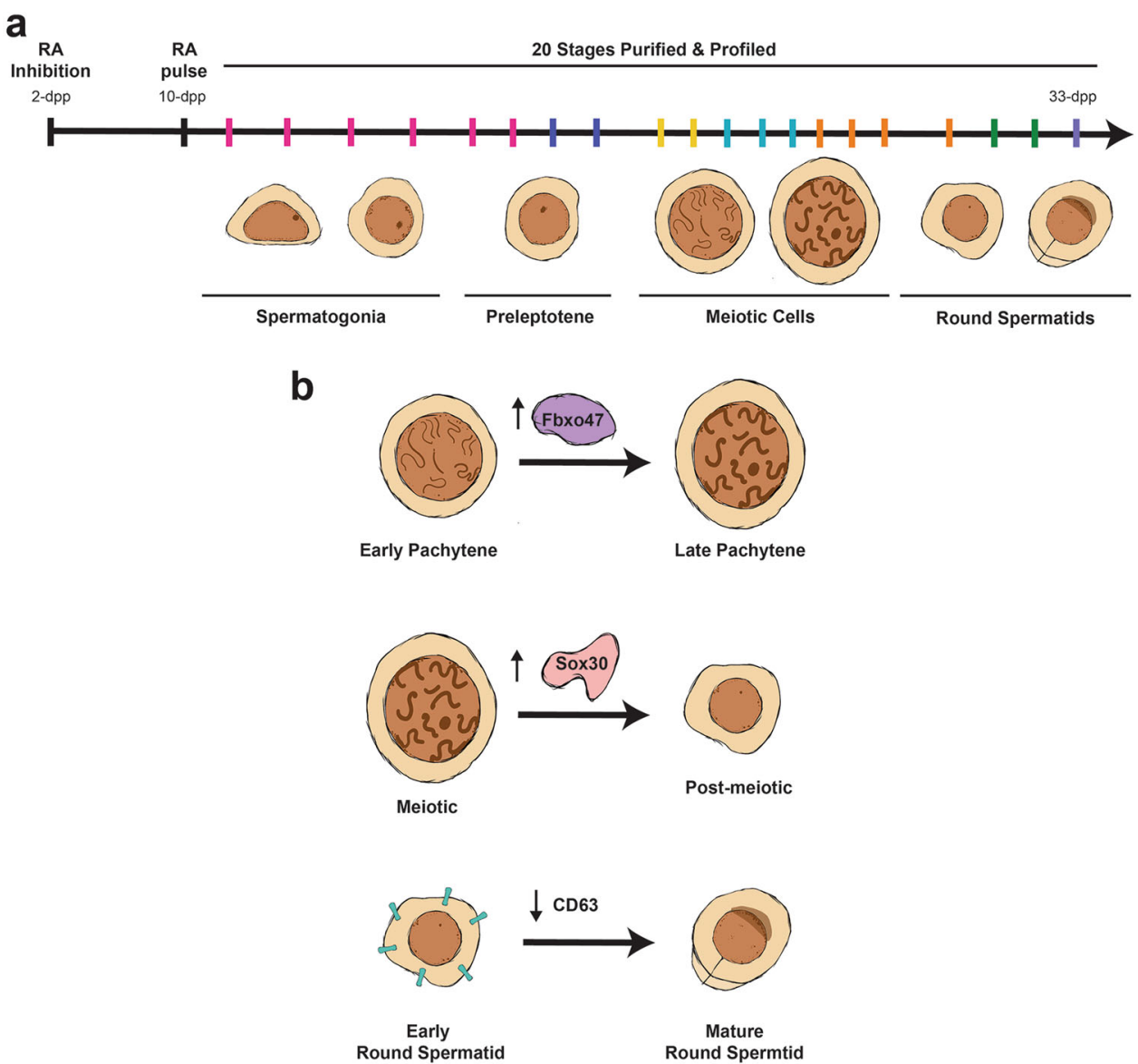

Fig. 1 Profiling spermatogenesis to identify newfound regulators. a Mice at 2 dpp were treated with a Retinoic Acid (RA) synthesis inhibitor for 7 days, followed by a RA pulse at $10 \mathrm{dpp}$ to stimulate the first wave of spermatogenesis. Dense time point collection sampled 20 stages of sperm development up till $32.5 \mathrm{dpp}$. Colors along the timeline represent the seven scRNA-seq t-SNE clusters obtained in the analysis. $\mathbf{b}$ Regulators identified in the analysis include, top: Fbxo47 in regulating recombination during meiosis, middle: transcription factor Sox30 in regulating spermatid development, bottom: cell surface marker CD63 in marking immature round spermatids

$\mathrm{CD}^{+} 3^{+}$spermatids. If the same markers are present in humans, then this would provide a new approach for selecting late round spermatids to aid more successful ROSI in the clinic. ${ }^{8}$

The study also revealed dynamic gene regulation signatures of developing sperm cells in the dataset. In male meiosis, the paired $X$ and $Y$ chromosomes are transcriptionally silenced due to unsynapsed regions. The authors confirmed that the silencing happens, but identified potentially stable sex chromosome transcripts persisting during this period, which requires further work in the future. The authors also confirmed that the haploid spermatids share the same levels of $X$ and $Y$ chromosome mRNA, despite only possessing one sex chromosome, supporting previous work that spermatids share transcripts via cytoplasmic bridges. $^{9}$

The data represent a detailed resource in the gene expression profiles of various stages of spermatogenesis, including lowabundant sub-populations, which may allow the identification and validation of more regulators in the future. For example, the resource could aid current work on spermatogenesis in vitro, for comparison with the expression profiles of the stages of spermatogenesis described in this study, and for addressing some aspects of infertility.

The combination of the gene expression profiles described, together with additional epigenomics characterization of sperm development, would be worthy of consideration. This might reveal the regulatory principles underlying the dynamic expression profiles seen. The outcomes from other similar ongoing studies would also be important for the field, especially as elongating spermatids and the surrounding niche of somatic subtypes were not profiled in this study. ${ }^{10,11}$

In summary, the study shows a careful application of scRNA-seq together with synchronization of developing cells that enriches rare cellular sub-populations. Such an approach can be used to identify and validate novel players during differentiation in other contexts.

\section{REFERENCES}

1. Amann, R. P. J. Androl. 29, 469-487 (2008).

2. Chen, Y. et al. Cell Res. 28, 879-896 (2018).

3. Clermont, Y. Physiol. Rev. 52, 198-236 (1972).

4. Picelli, S. et al. Nat. Methods 10, 1096-1098 (2013).

5. Lukassen, S., Bosch, E., Ekici, A. B. \& Winterpacht, A. Sci. Rep. 8, 1274-1277 (2018).

6. Snyder, E. M., Small, C. \& Griswold, M. D. Biol. Reprod. 83, 783-790 (2010).

7. Feng, C.-W. A. et al. Sci. Rep. 7, 17619 (2017).

8. Tanaka, A. et al. Proc. Natl Acad. Sci. USA 112, 14629-14634 (2015).

9. Braun, R. E., Behringer, R. R., Peschon, J. J., Brinster, R. L. \& Palmiter, R. D. Nature 337, 373-376 (1989).

10. Ernst, C., Eling, N., Martinez-Jimenez, C. P., Marioni, J. C. \& Odom, D. T. bioRxiv 2018. https://doi.org/10.1101/350868

11. Jung, M., Wells, D., Rusch, J., Ahmed, S. \& Marchini, J. bioRxiv 2018. https://doi.org/ $10.1101 / 393769$ 